1 Fundação Oswaldo Cruz (Fiocruz), Escola Nacional de Saúde Pública Sergio Arouca (Ensp) - Rio de Janeiro (RJ), Brasil. clabocage@hotmail.com

2 Fundação Oswaldo Cruz (Fiocruz), Escola Nacional de Saúde Pública Sergio Arouca (Ensp) - Rio de Janeiro (RJ), Brasil. claudia.osorio@ensp. fiocruz.br

\section{Gestão da Assistência Farmacêutica e demandas judiciais em pequenos municípios brasileiros: um estudo em Mato Grosso do Sul}

\author{
Pharmaceutical Services and litigation in small Brazilian \\ municipalities: a study in Mato Grosso do Sul
}

Cláudia Du Bocage Santos Pinto', Claudia Garcia Serpa Osorio-de-Castro²

\begin{abstract}
RESUMO O objetivo do estudo foi caracterizar a gestão da Assistência Farmacêutica, frente ao cenário da judicialização, em municípios de Mato Grosso do Sul. Foram visitadas as 27 comarcas de segunda entrância, e as informações referentes aos processos judiciais que demandavam medicamentos foram coletadas. Foram selecionados seis municípios para caracterizar a gestão da Assistência Farmacêutica através de entrevistas em profundidade com gestores. Foram identificadas deficiências graves nas atividades da Assistência Farmacêutica, possivelmente consolidando os problemas advindos dos pleitos judiciais, contrariando o que era esperado. Estima-se, assim, que a gestão incipiente da Assistência Farmacêutica nos municípios seria o principal determinante para o incremento das demandas.
\end{abstract}

PALAVRAS-CHAVE Assistência Farmacêutica; Direito à saúde; Preparações farmacêuticas; Sistema Único de Saúde.

ABSTRACT This study aimed to characterize management of Pharmaceutical Services in the scenario of judicial demands for medicines in municipalities of Mato Grosso do Sul. The twentyseven second-grade judicial districts in the State were visited and data pertaining to lawsuits were collected. Six municipalities were selected for characterization of Pharmaceutical Services through in-depth interviews with municipal managers. Serious problems in Pharmaceutical Services were observed, possibly adding to difficulties linked to judicial demands. Contrary to what was expected, basic failure in Pharmaceutical Services is the possible main determinant for the increase in litigation in these municipalities.

KEYWORDS Pharmaceutical Services; Right to health; Pharmaceutical preparations; Unified Health System. 


\section{Introdução}

Os entes federativos possuem farto arcabouço regulador para a gestão da Assistência Farmacêutica (AF), e sua gestão descentralizada está amplamente normatizada na legislação. Ao longo dos últimos anos, as deficiências da gestão na provisão de serviços e insumos, que culminam com a não disponibilização de medicamentos necessários à população, vêm contribuindo para o agravamento das condições de saúde dos indivíduos. Além disso, dão espaço para demandas judiciais, que agravam os problemas da gestão financeira de municípios e estados, em um efeito cíclico (MACHADO ET AL., 2011; ROMERO, 2008).

Diversos estudos revisaram problemas e distorções da gestão relacionados às demandas judiciais por medicamentos. Entre eles, pode-se destacar: má gestão da AF (PEPE ET AL., 2010B; VIEIRA; ZUCCHI, 2007); acesso a medicamentos desprovidos de evidências de segurança, eficácia ou efetividade (VIEIRA; ZUCCHI, 2007; MARQUES; DALLARI, 2007; PEREIRA ET AL., 2010); utilização da via judicial como ferramenta de acesso não igualitário (MACHADO ET AL., 2011); pressão imposta pela indústria farmacêutica para demanda de novos medicamentos (CHIEFFI; BARATA, 2010); e problemas relacionados à prescrição médica e à necessidade de revisão das listas e protocolos clínicos (PEPE ET AL., 2010B; VIEIRA; ZUCCHI, 2007).

Em que pese suas consequências, as demandas propiciaram que se tornasse mais evidente o panorama da provisão pública de medicamentos pelo Sistema Único de Saúde (SUS), e da gestão da AF, inclusive aquela exercida pelos entes municipais.

O crescente número de ações contribuiu para uma maior desorganização dos serviços de saúde em estados e municípios, causando grande impacto sobre a gestão e sobre o orçamento dos entes públicos (ROMERO, 2008; DELVECHIO, 2010). Já existem relatos na literatura sobre altos gastos provocados pelas ações judiciais de medicamentos impetradas contra entes estaduais e municipais, que se veem obrigados a remanejar o orçamento da AF para o atendimento às demandas (CASTRO, 2011). Em alguns estados e municípios foi necessário montar uma estrutura capaz de manejá-las tempestivamente (PEPE ET AL., 2010A).

O estado de Mato Grosso do Sul, situado na região Centro-Oeste do País, possui cerca de 2,5 milhões de habitantes, sendo $3 / 4$ destes inteiramente dependentes do SUS, já que apenas $1 / 4$ da população possui cobertura de plano de saúde privado (IBGE, 2009). De seus 78 municípios, a maioria é de pequeno porte apenas 4 deles possuem mais de 100 mil habitantes. Entre os demais, 73 possuem menos de 50 mil habitantes (IBGE, 2009).

O presente estudo focou a situação dos municípios de Mato Grosso do Sul com o objetivo de caracterizar a situação da gestão da $\mathrm{AF}$, frente às demandas judiciais de medicamentos.

\section{Métodos}

Trata-se de um estudo descritivo, de desenho transversal. Foi realizado em duas etapas, que, de modo a atender aos objetivos do estudo, investigou, na primeira, o panorama das demandas judiciais, e na segunda, a gestão da AF nos municípios.

$\mathrm{Na}$ primeira etapa, executada no ano de 2011, foram coletados dados sobre as demandas judiciais por medicamentos diretamente nos processos judiciais relativos aos anos de 2008 a 2011 depositados nas 27 comarcas de segunda entrância do estado de Mato Grosso do Sul. Estas foram eleitas por duas características: (i) por congregarem maior número de demandas judiciais do que as comarcas de primeira entrância, (ii) por incluírem municípios com estimada menor capacidade de gestão e recursos disponíveis, em relação aos municípios de comarca de entrância especial (maiores e mais populosos). Os dados foram coletados utilizando como referência o manual de indicadores para avaliação e monitoramento de demandas judiciais (PEPE, 2011). 
A segunda etapa, ocorrida no ano de 2012, foi iniciada pela seleção dos municípios para investigação da gestão municipal de AF. Para tanto, foram analisados os dados dos municípios integrantes das comarcas de segunda entrância (43 municípios) no que diz respeito ao número de processos e de habitantes. As comarcas foram divididas em três grupos, de acordo com o contingente populacional: pequeno (faixa $1-$ municípios com menos de 20 mil habitantes); médio (faixa 2 - municípios entre 20 mil e 50 mil habitantes); e grande (faixa $3-\mathrm{mu}$ nicípios acima de 50 mil habitantes). Em seguida, analisou-se o número de processos depositados em cada comarca no período de 2008 a 2011 e calculou-se a média de processos por habitante $(0,00075)$. As comarcas de contingente populacional 'médio' foram o maior grupo, representando cerca de $75 \%$ do total de comarcas de segunda entrância, razão pela qual foram priorizadas na seleção. Foram apontados os municípios-sede das comarcas e, dentre eles, seis foram selecionados. As identidades dos municípios foram preservadas, mediante substituição dos nomes por letras. Foram selecionados: (A), um município de faixa 1 e que possuía a maior relação demandas/habitante; (B), um município de faixa 3, com a menor relação demandas/habitante; quatro municípios de faixa 2 , sendo (C) o de maior relação demandas/habitante e (D) o de menor relação demandas/habitante no estrato superior da faixa, e (E) o de maior relação demandas/habitante e (F) o de menor relação demandas/habitante no estrato inferior da faixa.

Nesta etapa foi realizada uma investigação mais aprofundada da gestão da $\mathrm{AF}$. Foram feitas entrevistas com os gestores por meio de instrumento com questões semiabertas, baseado em 49 critérios objetivos enunciados como indicadores e organizados em dois componentes, referentes a: Gestão da AF (Recursos Humanos - 6 critérios; Instrumentos de Gestão - 6; Coordenação da
AF - 9; Atividades da AF - 22) e Demandas Judiciais (6 critérios).

Esses indicadores foram baseados no modelo lógico da $\mathrm{AF}$, já consolidado pela literatura (OPAS, 2005). Este permite verificar o encadeamento das ações, que devem conduzir a resultados esperados, tais como o adequado manejo de medicamentos, a segurança dos pacientes, e uma gestão eficiente, que redunde também nos resultados esperados. Sua utilização permite verificar como se dá a gestão da AF e em que pontos ela é tensionada em função das demandas judiciais por medicamentos, que ocorrem nos municípios investigados.

Ao final, as respostas foram tabuladas de forma a responder objetivamente aos indicadores.

A seguir, foi feito um consenso de especialistas, realizado com base no método Delfos (Delphi) (WRIGHT; GIOVINAZZO, 2000). Foram convidados profissionais da gestão e da judicialização na área de saúde, totalizando três especialistas. Eles classificaram os resultados dos indicadores sobre AF de cada um dos seis municípios, com o auxílio de uma escala arbitrária de valor, de modo a definir um diagnóstico para cada município. Foram atribuídas três graduações a cada indicador: resposta apropriada no âmbito da gestão; parcialmente apropriada; ou inapropriada e com consequências potencialmente danosas à gestão da $\mathrm{AF}$. O melhor desempenho correspondeu ao maior número de critérios apropriados.

Após essa classificação, os resultados foram analisados de duas formas: (i) adequação de todos os componentes, por município; e (ii) adequação de todos os municípios, por componente, e testados para significância estatística pelo $\chi^{2}$ de Pearson (Abramson JH, WinPepi versão 11.44, 2015).

Os dados de execução financeira informados pelos gestores foram complementados por pesquisa em bases de dados secundárias da Secretaria Estadual de Saúde de Mato Grosso do Sul e do Ministério da Saúde. 
A execução foi parcialmente subsidiada pela Coordenação de Aperfeiçoamento de Pessoal de Nível Superior (Capes), através da Bolsa de Produtividade concedida a uma das autoras. Não houve qualquer conflito de interesses na execução do trabalho.

O estudo foi aprovado pelo Comitê de Ética em Pesquisa da Escola Nacional de Saúde Pública Sergio Arouca sob o número de parecer 237/11 (CAAE: 0252.0031.00011). Todos os gestores aceitaram participar por meio de Termo de Consentimento Livre e Esclarecido (TCLE).

\section{Resultados}

\section{Demandas judiciais}

O levantamento de processos através das certidões solicitadas às comarcas indicou um total de 6.100 processos, nos quais os municípios constavam como réus das ações. A análise posterior de cada um dos processos, através do site do Tribunal de Justiça de Mato Grosso do Sul (TJMS), trouxe a informação de que, desse total, $1.825(30 \%)$ eram processos relativos às demandas de saúde. Dentro desse segundo grupo, 771 (42\%) eram processos de demandas por medicamentos. Observou-se um aumento de $47 \%$ no número total de processos entre 2008 e 2011.

A maioria das ações (62\%) foi representada pela Defensoria Pública Estadual. A maioria dos pedidos de antecipação de tutela (82\%) foi julgada como favorável ao demandante. Em poucas ações foi solicitada a manifestação dos réus antes do julgamento (18\%). O estado figurou como réu solidário ao município na maioria das ações (58\%).

No que diz respeito aos medicamentos pleiteados, foi observado grande percentual de pedidos de medicamentos para tratamento de condições que envolvem o sistema cardiovascular (25\% do total de pleitos) e o sistema gastrointestinal (11\%). Para os municípios analisados nas questões referentes à gestão da $\mathrm{AF}$, não houve nenhum pedido de medicamento sem registro na Agência Nacional de Vigilância Sanitária (Anvisa). No entanto, foram observados pleitos por medicamentos com indicação de uso off-label no município A (17\%), no município D (11\%) e no município E (9\%). Foi observado ainda que, com exceção do município D (com 49\%), mais da metade dos medicamentos demandados via justiça não fazia parte de nenhum dos componentes de financiamento da AF. Nos municípios B e D, mais de $40 \%$ dos medicamentos demandados pertenciam ao componente da Atenção Básica (AB).

\section{Gestão da Assistência Farmacêutica}

O quadro 1 apresenta os principais resultados do estudo nos municípios, destacando as possíveis relações entre as demandas judiciais e a AF neles praticada.

Como resultados comuns, pode-se destacar ainda que em nenhum dos municípios houve demanda judicial por medicamentos do Componente Estratégico; todos os municípios, com exceção do município B, relataram que a logística desses medicamentos era gerida fora da $\mathrm{AF}$.

As Informações da Vigilância Epidemiológica (VE) não eram utilizadas para embasar ações da AF em nenhum dos municípios. Os médicos municipais não possuíam capacitação para prescrição segundo Uso Racional de Medicamentos (URM).

Nenhum município possuía estrutura de transporte específica para medicamentos. Não havia uma rotina de verificação periódica de estruturas e das atividades, pela gestão da AF. Todos os municípios relataram que a programação se baseava exclusivamente no consumo histórico, e que as compras levavam em conta apenas o critério de preço, sem utilizar nenhuma ferramenta de gestão para auxílio da atividade. 


\begin{tabular}{|c|c|c|}
\hline \multirow[b]{2}{*}{ Município } & \multicolumn{2}{|c|}{ Componentes } \\
\hline & Demandas judiciais e gestão & Gestão da AF \\
\hline$A$ & $\begin{array}{l}\text { Medicamentos demandados presentes na Relação Nacional de } \\
\text { Medicamentos Essenciais (Rename): } 29 \% \\
\text { Medicamentos demandados para uso off-label: 17\% } \\
\text { Medicamentos demandados do componente da AB: 21\% } \\
\text { Medicamentos demandados do componente especializado: 25\% } \\
\text { Medicamentos demandados ausentes dos componentes de } \\
\text { financiamento: } 54 \% \\
\text { Demandas judiciais são resolvidas em âmbito administrativo. Os } \\
\text { medicamentos demandados são manejados junto aos demais } \\
\text { medicamentos comprados e fornecidos pelo município, geridos } \\
\text { pelo mesmo farmacêutico e incluídos na programação e no } \\
\text { processo licitatório. }\end{array}$ & $\begin{array}{l}\text { Não há Comissão de Farmácia e Terapêutica (CFT) nem Relação } \\
\text { Municipal de Medicamentos (Remume). Município adota os } \\
\text { Protocolos Clínicos e Diretrizes Terapêuticas (PCDT). } \\
\text { Há um único farmacêutico atuante na gestão e na Central de } \\
\text { Abastecimento Farmacêutico (CAF). } \\
\text { Não há farmacêuticos atuando na dispensação das unidades. } \\
\text { Existe Plano Municipal de Saúde, mas não contempla a AF. } \\
\text { A lista de medicamentos do município é baseada na lista pactuada } \\
\text { na Comissão Intergestores Bipartite (CIB). Há uma lista de } \\
\text { medicamentos não pactuados, composta por } 97 \text { itens. Dentre estes, } \\
\text { muitos se encontram fora da Rename. } \\
\text { O farmacêutico tem atuação limitada no processo de compras, que } \\
\text { se dá por licitação, à exceção das emergenciais. } \\
\text { Atividades logísticas e dispensação são deficientes e não seguem } \\
\text { normas técnicas. }\end{array}$ \\
\hline B & $\begin{array}{l}\text { Medicamentos demandados presentes na Rename: 39\% } \\
\text { Medicamentos demandados para uso off-label: 0\% } \\
\text { Medicamentos demandados do componente da AB: } 42 \% \\
\text { Medicamentos demandados do componente especializado: 6\% } \\
\text { Medicamentos demandados ausentes dos componentes de } \\
\text { financiamento: 52\% } \\
\text { Não há procedimento específico instituído para o manejo das } \\
\text { demandas judiciais. Estas são geridas totalmente em separado } \\
\text { dos demais medicamentos fornecidos pelo município. Não há } \\
\text { análise técnica dos pedidos. Os medicamentos não são incluídos } \\
\text { na programação, nem são comprados por licitação. Ficam } \\
\text { armazenados no setor jurídico e são entregues por um advogado. }\end{array}$ & $\begin{array}{l}\text { Não há CFT nem Remume. Município não adota os PCDT. } \\
\text { Há apenas um farmacêutico no nível central, sem capacitação } \\
\text { específica para gestão. } \\
\text { Não há farmacêuticos na CAF nem nas unidades de dispensação. } \\
\text { Existe Plano Municipal de Saúde, mas não contempla a AF. } \\
\text { Cerca de } 90 \% \text { dos medicamentos da lista do município dizem } \\
\text { respeito aos pactuados na CIB. A lista é revista uma vez por ano e } \\
\text { elaborada pelo farmacêutico. } \\
\text { O farmacêutico não participa do processo de compras, que é feito } \\
\text { por licitação. } \\
\text { Atividades logísticas e dispensação são deficientes e não seguem } \\
\text { normas técnicas. }\end{array}$ \\
\hline$C$ & $\begin{array}{l}\text { Medicamentos demandados presentes na Rename: 13\% } \\
\text { Medicamentos demandados para uso off-label: } 0 \% \\
\text { Medicamentos demandados do componente da AB: 6\% } \\
\text { Medicamentos demandados do componente especializado: 6\% } \\
\text { Medicamentos demandados ausentes dos componentes de } \\
\text { financiamento: } 88 \% \\
\text { Não há um procedimento formal instituído para o manejo dos } \\
\text { medicamentos demandados, que são geridos à parte dos demais. } \\
\text { Ficam sob a responsabilidade de um advogado, na Secretaria Municipal } \\
\text { de Saúde (SMS). Não ocorre análise técnica do pedido. Alguns são } \\
\text { incluídos na programação, mas a compra é feita em farmácias privadas. }\end{array}$ & $\begin{array}{l}\text { Não há CFT nem Remume. Município não adota os PCDT. } \\
\text { Há um farmacêutico no nível central capacitado para gestão, que } \\
\text { atua também na CAF. } \\
\text { Não há farmacêutico em nenhuma das unidades de dispensação do } \\
\text { município. } \\
\text { Existe Plano Municipal de Saúde, mas não contempla a AF. } \\
\text { Utiliza a lista pactuada pelo estado, mas inclui } 83 \text { itens além dos } \\
\text { pactuados. Destes, } 70 \text { não pertencem a Rename, e são incluídos para } \\
\text { evitar demandas judiciais, uma vez que são continuamente prescritos } \\
\text { pelos médicos do município. } \\
\text { O farmacêutico realiza sozinho a seleção de medicamentos, e } \\
\text { participa do processo de licitação. } \\
\text { Atividades logísticas e dispensação são deficientes e não seguem } \\
\text { normas técnicas. }\end{array}$ \\
\hline $\mathrm{D}$ & $\begin{array}{l}\text { Medicamentos demandados presentes na Rename: } 46 \% \\
\text { Medicamentos demandados para uso off-label: } 11 \% \\
\text { Medicamentos demandados do componente da AB: } 40 \% \\
\text { Medicamentos demandados do componente especializado: 11\% } \\
\text { Medicamentos demandados ausentes dos componentes de } \\
\text { financiamento: } 49 \% \\
\text { As demandas judiciais são responsabilidade do Departamento } \\
\text { de Assistência Social. Para evitar novas demandas, o município } \\
\text { instituiu uma 'via assistencial', por meio da qual são fornecidos } \\
\text { todos os medicamentos procurados pelos pacientes. } \\
\text { Os medicamentos demandados, de uso contínuo, são incluídos } \\
\text { na programação e passam a ser comprados por meio de licitação. } \\
\text { Nos demais casos, as compras são realizadas diretamente nas } \\
\text { farmácias privadas do município, para onde os pacientes se } \\
\text { dirigem diretamente, a fim de retirar o medicamento. }\end{array}$ & $\begin{array}{l}\text { Não há CFT nem Remume. Município não adota os PCDT. } \\
\text { Há um farmacêutico na coordenação da AF, mas sem capacitação } \\
\text { específica para gestão, sendo o mesmo profissional responsável } \\
\text { pela CAF. } \\
\text { Das sete unidades de saúde do município, em apenas uma há } \\
\text { farmacêutico. } \\
\text { O Plano Municipal de Saúde contempla a AF e a coordenação de } \\
\text { AF está inserida no organograma da SMS. } \\
\text { A lista de medicamentos é elaborada pelo farmacêutico, baseando- } \\
\text { se na lista pactuada, e acrescentando medicamentos, sendo que } \\
\text { alguns não integram a Rename. } \\
\text { O farmacêutico não participa do processo de compras. } \\
\text { Atividades logísticas e dispensação são deficientes e não seguem } \\
\text { normas técnicas. }\end{array}$ \\
\hline
\end{tabular}




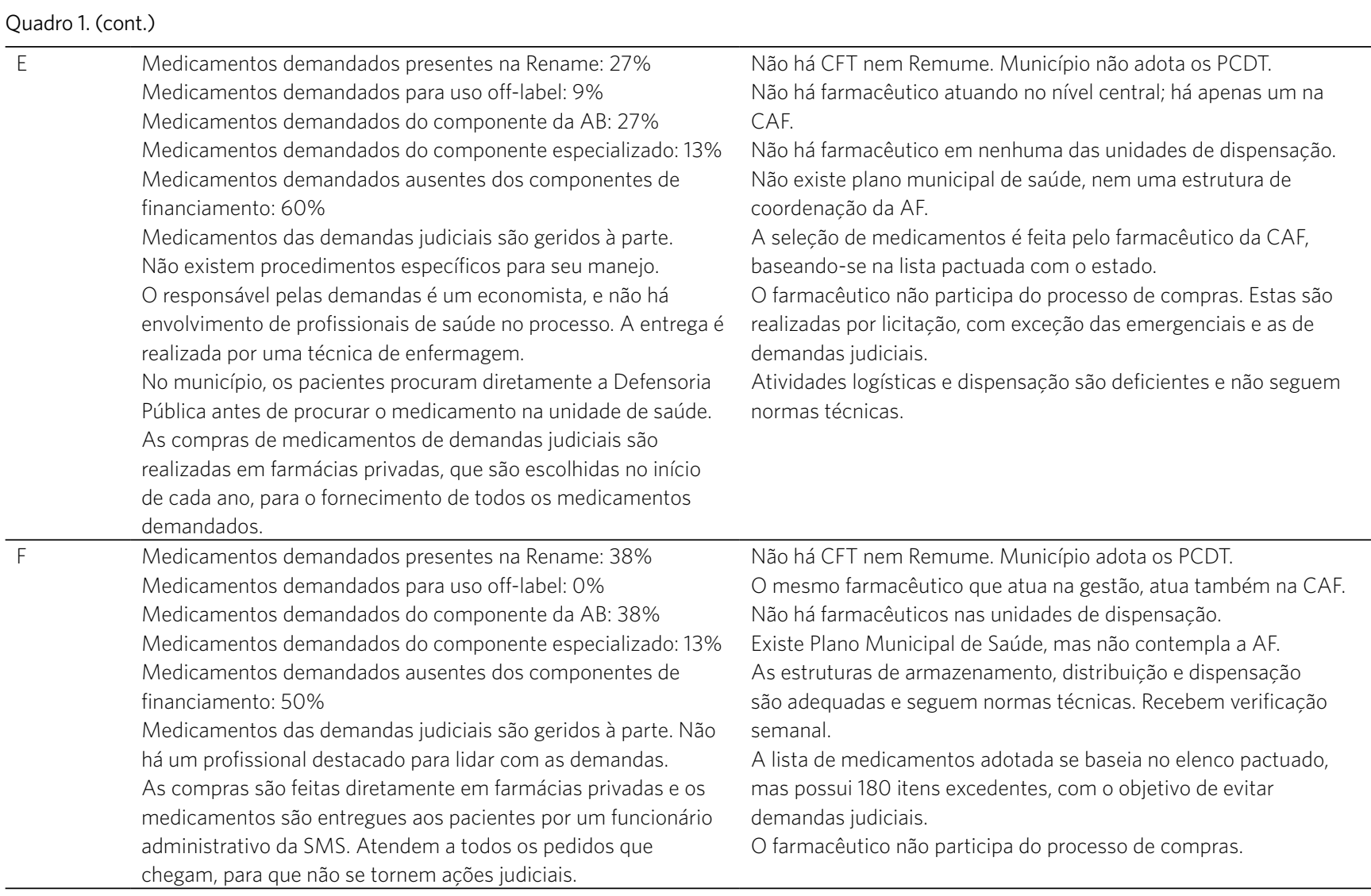

O consenso de especialistas observou os resultados dos indicadores sob o olhar da gestão - em última análise, o tipo de consequência provocada pela adequação, ou inadequação, em cada critério dos componentes da gestão da AF (1. Recursos Humanos; 2. Instrumentos de Gestão; 3. Coordenação da AF; 4. Atividades da AF) e das demandas judiciais (5. Demandas Judiciais), em cada município (A, B, C, D, E e F), como se vê na tabela 1.

Tabela 1. Contagem de critérios da gestão da Assistência Farmacêutica, de acordo com classificação dos especialistas, por município. Municípios selecionados, Mato Grosso do Sul, 2012

\begin{tabular}{lccc}
\hline & \multicolumn{3}{c}{ Número de critérios } \\
\cline { 2 - 4 } Municípios & Adequados & Parcialmente adequados & Inadequados \\
\hline A & 16 & 11 & 22 \\
B & 15 & 11 & 23 \\
C & 9 & 12 & 28 \\
D & 10 & 10 & 29 \\
E & 9 & 8 & 32 \\
F & 12 & 13 & 24 \\
TOTAL & 71 & 65 & 158 \\
\hline
\end{tabular}

Fonte: Elaboração própria. 
Pôde ser observada a maior quantidade de resultados inadequados em todos os municípios estudados. O município E, em números absolutos, apresentou os piores resultados, com mais de $67 \%$ dos indicadores relacionados à gestão da $\mathrm{AF}$ classificados como inadequados. No entanto, a análise estatística não determinou diferenças significativas entre os municípios, com relação a melhores ou a piores desempenhos.

A seguir, foi realizada a análise por critério, em todos os municípios (tabela 2).

Tabela 2. Contagem de critérios da gestão da Assistência Farmacêutica, de acordo com classificação dos especialistas, por componente. Municípios selecionados, Mato Grosso do Sul, 2012

\begin{tabular}{lccc}
\hline & \multicolumn{3}{c}{ Número de critérios } \\
\cline { 2 - 4 } Componentes & Adequados & $\begin{array}{c}\text { Parcialmente } \\
\text { adequados }\end{array}$ & Inadequados \\
\hline Assistência Farmacêutica & & & 23 \\
Recursos Humanos & 1 & 12 & 14 \\
Instrumentos de Gestão & 13 & 9 & 20 \\
Coordenação da AF & 27 & 7 & 79 \\
Atividades da AF* & 21 & 32 & 23 \\
Demandas Judiciais & 8 & 5 & 159 \\
TOTAL & 70 & 65 & \\
\hline
\end{tabular}

Fonte: Elaboração própria.

Nota: ${ }^{\star} \mathrm{P}=0,007$.

De modo geral, nos critérios Recursos Humanos e Atividades da AF, houve muito mais indicadores julgados inadequados do que adequados. O critério que melhor se posicionou foi o relacionado à coordenação da AF. O critério com pior desempenho entre todos os municípios foi o 4, correspondente às Atividades da AF. Nele, houve diferença significativa em relação ao pior e ao melhor desempenho nos municípios.
A tabela 3 é referente às informações financeiras obtidas nas entrevistas com os gestores municipais. Os municípios A e E não informaram as despesas relativas aos medicamentos do Componente Básico da AF. Apenas no município $\mathrm{C}$ não foi fornecida a informação a respeito das despesas com demandas judiciais de medicamentos. Nos municípios B e E não foram fornecidas informações sobre despesas com medicamentos não pactuados.

Tabela 3. Valores informados pelos municípios selecionados, referentes às despesas com medicamentos. Mato Grosso do Sul, 2011

\begin{tabular}{|c|c|c|c|c|c|c|}
\hline \multirow{2}{*}{$\begin{array}{l}\text { Despesas municipais com } \\
\text { medicamentos (em } \mathrm{R} \$ \text { ) }\end{array}$} & \multicolumn{6}{|c|}{ Município } \\
\hline & A & B & C & D & $\mathbf{E}$ & $\mathbf{F}$ \\
\hline AF básica & Não informado & $161.612,64$ & $334.000,00$ & $38.342,91$ & Não informado & $199.211,97$ \\
\hline Demandas judiciais & $10.200,00$ & $9.212,86$ & Não informado & $8.277,00$ & $363.030,48$ & $70.412,36$ \\
\hline Não pactuados & $90.000,00$ & Não informado & $60.612,00$ & $63.017,84$ & Não informado & $84.000,00$ \\
\hline TOTAL & $x$ & $x$ & $x$ & $109.637,75$ & $x$ & $353.624,33$ \\
\hline
\end{tabular}

Fonte: Elaboração própria a partir de dados de entrevistas com gestores municipais. 
Verificou-se que, em todos os municípios, além do valor aportado para o Componente Básico da AF, os entes municipais aportavam ainda montantes extras para medicamentos de demanda judicial e medicamentos não pactuados.

O município $\mathrm{F}$ aportou $56 \%$ do gasto total de medicamentos para a AF básica, $24 \%$ para medicamentos não pactuados e quase $20 \%$ para medicamentos de demandas judiciais. O município D aportou $35 \%$ do gasto total de medicamentos para a $\mathrm{AF}$ básica, $57 \%$ para medicamentos não pactuados e $0,8 \%$ para medicamentos de demandas judiciais. Nos municípios A, B, C e E faltam uma ou mais variáveis que permitiriam uma análise relacional dos gastos.

A tabela 4 faz uma comparação dos valores per capita gastos pelo município para cobertura dos medicamentos do Componente Básico da AF, das demandas judiciais e dos medicamentos não pactuados. A primeira coluna fornece a informação sobre o valor per capita mínimo, estipulado pela Portaria $n^{\circ} 4.217$, de dezembro de 2010, vigente à época do estudo.

Tabela 4. Investimentos per capita para cobertura de medicamentos do Componente Básico da Assistência Farmacêutica, de demandas judiciais e não pactuados. Municípios selecionados, Mato Grosso do Sul, 2011

\begin{tabular}{lcccc}
\hline & \multicolumn{4}{c}{ Investimento per capita } \\
\cline { 2 - 5 } Municípios & Mínimo pactuado* & $\begin{array}{c}\text { Aportado para } \\
\text { Componente Básico } \\
\text { da AF }\end{array}$ & Demandas judiciais & $\begin{array}{c}\text { Medicamentos não } \\
\text { pactuados }\end{array}$ \\
\hline A & 1,86 & - & 0,76 & 6,72 \\
B & 1,86 & 1,58 & 0,09 & - \\
C & 1,86 & 15,68 & - & 2,84 \\
D & 1,86 & 1,96 & 0,42 & 3,20 \\
E & 1,86 & - & 11,42 & - \\
F & 1,86 & 4,82 & 1,70 & 2,03 \\
\hline
\end{tabular}

Fonte: Elaboração própria através de dados de entrevistas com gestores municipais. Nota: *De acordo com a Portaria no 4.217, de dezembro de 2010.

Os aportes para o Componente Básico da AF variaram de $\mathrm{R} \$ 1,58$ per capita em $\mathrm{B}$ até $\mathrm{R} \$$ 15,68 per capita em C. No caso das demandas judiciais, a variação foi de $\mathrm{R} \$ 0,09$ per capita em $\mathrm{B}$, até $\mathrm{R} \$ 11,42$ per capita em E. No caso de medicamentos não pactuados, $\mathrm{A}$ foi o município com maior valor per capita aportado, $\mathrm{R} \$ 6,72$.

Entre aqueles que forneceram informações sobre o Componente Básico - responsabilidade municipal - três (C, D e F) gastavam mais per capita que o mínimo estipulado na legislação vigente.

\section{Discussão}

O estudo mostrou que as demandas em 27 comarcas de segunda entrância no MS cresceram entre 2008 e 2011 . O estudo nos municípios selecionados mostrou muitas dificuldades da AF, por falta de estrutura ou inconformidades, e também que não houve diferenças significativas da gestão da $\mathrm{AF}$ entre os municípios. Não foi surpreendente, assim, constatar que o componente de gestão, que mostrou pior desempenho em todos os municípios, foi aquele referente às atividades da AF.

O crescente número de demandas ao longo dos anos é coerente com o que vem ocorrendo nos demais estados do País, como demonstrado no estudo realizado em Santa Catarina, onde o número de processos saltou de 2, em 2000, para 444, em 2004 (PEREIRA ET AL., 2010). E é igual à tendência de deferimento dos pleitos, como demonstrado no estudo 
realizado no Rio de Janeiro, em 2006, onde, dos 185 processos analisados apenas três tiveram os pedidos negados (PEPE ET AL., 2010B).

A investigação aprofundada em municípios selecionados, com diferentes relações entre número de processos e habitantes, possibilitou análise da gestão da $\mathrm{AF}$ em distintos cenários frente às demandas judiciais. O julgamento realizado pelos especialistas ajudou a apontar os logros e as deficiências das gestões municipais, sendo o componente de atividades da AF o percebido como mais problemático.

No município A, os medicamentos de demandas judiciais eram manejados junto aos demais medicamentos fornecidos pelo município. Observou-se alto percentual de medicamentos demandados não integrantes dos componentes de financiamento da AF; houve também medicamentos demandados prescritos para uso off-label. As estruturas e os recursos humanos eram deficientes, assim como as atividades da AF. A ausência de Comissão de Farmácia e Terapêutica (CFT), assim como a falta de profissionais capacitados e atualizados com as normativas e protocolos pode ter influenciado este cenário. Em B havia diversas deficiências, que podem ter influenciado a ocorrência das demandas. No município houve um percentual considerável de demandas por medicamentos da $\mathrm{AB}$ (42\%) e de medicamentos integrantes da Relação Nacional de Medicamentos Essenciais (Rename) (39\%). A AF gerida de forma ineficiente pode ter levado à indisponibilidade de medicamentos, seja por seleção inadequada, programação insuficiente ou logística precária.

Em C, chamou a atenção o alto percentual (88\%) de demandas por medicamentos não pertencentes aos componentes de financiamento da AF, apesar de a lista de medicamentos do município possuir 83 itens além dos pactuados, justamente incluídos com o intuito de evitar as demandas. A pressão das demandas distorceu o processo de inclusão na lista, a partir do momento em que o município não realizou seleção. Situação semelhante, porém, mais aguda, ocorreu no município $\mathrm{F}$, que adotou como estratégia a inclusão de 180 medicamentos além daqueles pactuados com o estado para evitar demandas. Problemas de base, principalmente de deficiência e capacitação de recursos humanos, impediram que o diferencial proporcionado pela estrutura e pelas atividades logísticas oferecessem ganho no enfrentamento da judicialização.

Em D prevaleceu o fornecimento de medicamentos por via assistencial, na qual os pacientes, apenas munidos da prescrição, obtinham os medicamentos de que necessitavam. Essa via surgiu, provavelmente, com o intuito de evitar a via judicial, mas expôs as falhas da AF municipal e a falta de normas municipais mínimas para o fornecimento de medicamentos.

$\mathrm{O}$ cenário mais crítico da gestão da $\mathrm{AF}$ frente às demandas foi encontrado no município E. A compra de medicamentos em farmácia privada, escolhida para fornecê-los para o município, sugere total falta de normas e procedimentos técnicos. Não é de se estranhar, portanto, que os pacientes, possivelmente habituados ao desabastecimento de medicamentos, sequer procurassem as unidades de saúde, dirigindo-se diretamente à Defensoria Pública.

A falta de estrutura encontrada em muitas secretarias estaduais e municipais de saúde já foi apontada por diversos estudos como um fator importante para o crescimento das demandas judiciais (ROMERO, 2008; PEREIRA, 2010). As baixas estruturas operacional, logística e de atendimento já se impõem como entraves à execução das atividades regulares da gestão e do ciclo da AF, quiçá para a assunção de novas responsabilidades impostas pelas demandas.

A questão dos recursos humanos foi crítica em todos os locais, e esse é um problema corrente, já demonstrado em muitos municípios (VIEIRA, 2008; DE BERNERDI, 2006). A falta de profissionais capacitados se mostra em desacordo com a Política Nacional de Medicamentos, implica na má aplicação dos 
recursos públicos e no baixo retorno em efetividade na utilização dos medicamentos.

Atividades da AF sem embasamento em dados epidemiológicos podem implicar na ocorrência de demandas judiciais (FIGUEIREDO; PEPE; OSORIO-DE-CASTRO, 2010). A inexistência de uma CFT ou da Relação Municipal de Medicamentos (Remume) deixa uma lacuna importante nas atividades da AF dos municípios (WHO, 2003), uma vez que a falta de critérios para a elaboração de uma lista municipal ou para a programação dos medicamentos repercute na forma de um elenco incompleto e/ou no desabastecimento. Em todos os municípios, algum tipo de seleção era realizado, já que havia uma lista de medicamentos não pactuados, e, portanto, não relacionados à Rename. Pela falta dos instrumentos comuns à seleção, há que se considerar que as escolhas podem não ter sido as melhores ou mais seguras para a população.

A não adoção de Protocolo Clínico e Diretrizes Terapêuticas (PCDT) também é um fator diretamente relacionado à ocorrência das demandas. A falta de adesão aos protocolos propicia a prescrição de medicamentos inadequados à indicação ou fora do rol dos medicamentos previstos pelo SUS. A literatura mostra que, na maioria dos casos, a prescrição é prevalente sobre quaisquer outros argumentos da gestão (ROMERO, 2008; PEPE, 2010A).

A incorporação dos medicamentos de demandas judiciais na programação dos municípios é uma medida adotada com certa frequência em outros contextos (PEPE, 2010A). Com relação às compras, a situação se mostrou mais problemática, uma vez que todos os municípios relataram compras diretas em farmácias privadas, dando margem à falta de transparência e de economia para os cofres públicos (PEPE, 2010A). Apesar do relato da realização de compras regulares por meio de licitação, não houve adoção de ferramentas para auxílio nos processos, tais como catálogo de especificações técnicas dos medicamentos para os editais de aquisição, ou utilização do Banco de Preços em Saúde, retratando um processo de compras deficiente e com pouco ou nenhum respaldo técnico.
A falta de estrutura e de recursos humanos capacitados, além da ausência do farmacêutico no manejo das demandas pode ter impossibilitado a orientação aos pacientes nas unidades de dispensação. Assim como em outras realidades estudadas (ARAÚjo ET AL., 2008), percebe-se que a dispensação em unidades públicas do SUS fica limitada a funcionar como um simples local de entrega de medicamentos. A falta de informações sobre o desenvolvimento do tratamento pode colocar em risco a saúde do indivíduo (FIGUEIREDO; PEPE; OSORIO-DE-CASTRO, 2010). A despeito disto, é incomum que pacientes que recebam medicamentos pela via judicial sejam acompanhados pela gestão da AF.

Em relação à execução financeira, observou-se que nem todos os gestores sabiam, ou quiseram informar, dados de despesas com medicamentos. Se fossem considerados apenas os municípios D e F, para os quais há informações completas de gastos, poder-se-ia admitir que a estratégia de aumento das despesas com medicamentos não pactuados, operada proporcionalmente, em maior grau, pelo município $\mathrm{D}$, talvez resultasse em menores gastos com medicamentos de demandas judiciais.

Outros fatores, como aqueles relacionados à indicação e à prescrição, às características do uso off-label, às pressões de incorporação, cuja abordagem foge ao escopo deste trabalho, podem influenciar a ocorrência de demandas. O que se tem observado no País é o incremento do número de demandas e dos gastos dos entes federativos a elas relacionados, a despeito de quaisquer medidas de contenção (VIEIRA; ZUCCHI, 2007).

No entanto, não há como realizar uma análise integrada relacionando gastos da AF com demandas judiciais no conjunto de municípios, pois não há dados completos para todos. Não se sabe qual é o investimento per capita real dos municípios A, B, C e E. Observa-se que o gasto per capita em demandas judiciais do município E é alto em relação aos demais; sabe-se que, neste município, $60 \%$ das demandas são para medicamentos 
ausentes dos componentes de financiamento da $\mathrm{AF}$, o que poderia relacionar-se ao alto gasto com demandas. Por outro lado, o município $\mathrm{C}$, que possui o maior gasto per capita com medicamentos da $\mathrm{AB}$ ( $\mathrm{R} \$ 15,68)$, tem, consequentemente, apenas $6 \%$ das demandas relativas a este componente. Mas é também o que possui a maior proporção de demandas com medicamentos ausentes das listas de financiamento (88\%).

No tocante aos municípios nos quais todos os dados estão relatados, as informações também não permitem identificar relações. Os municípios D e F apresentam proporções muito semelhantes em relação ao tipo de medicamento demandado e ao tipo de medicamento demandado por componente de financiamento. O primeiro gasta mais com medicamentos não pactuados do que o segundo, o que poderia explicar o também maior percentual de uso off-label. Por outro lado, o município F gasta mais com AF básica do que o D. Este gasto, no entanto, parece não influenciar os gastos per capita com demandas judiciais.

A média do gasto per capita com medicamentos, de municípios com menor número de habitantes, tende a ser maior do que a de municípios mais populosos, segundo Vieira (2011). Uma provável maior participação de planos de saúde em municípios mais populosos direcionaria parcela importante da população para a aquisição privada de medicamentos em farmácias comerciais. E o menor poder de escala e de negociação dos pequenos municípios levaria à obtenção de preços mais altos nas compras de medicamentos (VIEIRA, 2011).

$\mathrm{O}$ aporte adicional de recursos para compra de medicamentos foi medida adotada pelos gestores como tentativa de resolução de problemas, e não pareceu significar um entrave para a gestão, nos municípios estudados. Além disso, todos os municípios relataram receber os aportes da esfera federal e da esfera estadual, conforme o pactuado.

O Pacto pela Gestão do SUS passou a prever financiamento específico para a gestão do sistema, em que os recursos devem ser voltados para apoiar iniciativas de fortalecimento da gestão. A própria Portaria $n^{0}$ 4.217, de dezembro de 2010, deixava clara a possibilidade de utilização de $15 \%$ dos recursos provenientes de estados e municípios para adequação de espaço físico das farmácias municipais, aquisição de equipamentos e mobiliário, e para realização de atividades voltadas para educação continuada e qualificação dos recursos humanos da AF.

Na questão da gestão do trabalho, o Pacto tem ainda como uma de suas diretrizes a política de recursos humanos. É eixo fundamental para o funcionamento do SUS, sendo as secretarias municipais e estaduais de saúde responsáveis por empreender esforços para a criação ou fortalecimento de estruturas de recursos humanos em seus quadros. Entretanto, não foi observada a apropriação dessas possibilidades pelos municípios estudados. Muitos não possuíam plano municipal de saúde contemplando a AF, ou coordenação formal de AF. Tampouco utilizavam ferramentas para auxílio na gestão. A falta de investimentos em recursos humanos e estruturais redunda também na falta de capacidade para utilização de ferramentas e cumprimento de determinações do SUS.

Ainda que seja um considerável determinante sobre a situação da AF, a gestão da saúde, como um todo, nos municípios, não foi analisada. Algumas informações relatadas pelos gestores, mormente relacionadas à logística de medicamentos, não puderam sofrer comprovação integral; houve grande dificuldade, em alguns municípios, de acesso aos dados financeiros. As possíveis causas dessa situação seriam a falta de capacitação técnica dos recursos humanos municipais para a gestão ou a falta de transparência praticada pela gestão municipal. Mas, quaisquer que sejam os motivos, tais situações, por si só, podem também ser consideradas como resultados que depõem contra a gestão nesses municípios.

Por fim, o perfil diferenciado de alguns municípios que apresentaram grande número de demandas judiciais merece 
análise mais aprofundada, para que possam ser compreendidas as causas específicas desta situação. Não foi possível, nos limites desta pesquisa, identificar tais razões.

O presente trabalho inova ao abordar a gestão da $\mathrm{AF}$ frente às demandas em pequenos municípios, uma vez que a maioria dos estudos realizados até hoje abordam estados ou grandes municípios (DE BERNERDI; BIEBERBACH THOMÉ, 2006; MACHADO, 2011; MARQUES, 2007; PEPE 2010B; PEREIRA, 2010). Destaca-se que, em relação a Mato Grosso do Sul, os municípios estudados não eram os menores do estado. Mas, se comparados à distribuição dos municípios no Brasil, seriam considerados pequenos, uma vez que o IBGE entende como de pequeno porte municípios com até 50 mil habitantes (IBGE, 2009). As questões estruturais e de recursos humanos encontradas assemelhavam-se àquelas presentes em outros pequenos municípios brasileiros (VIEIRA, 2008; DE BERNERDI; BIEBERBACH; THOMÉ, 2006).

\section{Conclusão}

O presente trabalho partiu do pressuposto, já demonstrado em outros estudos, de que as demandas judiciais têm impacto sobre a gestão da $\mathrm{AF}$, provocando aumento de gastos e desestruturação dos serviços. Neste, observou-se que a judicialização, em maior ou menor grau, acabou por impor certas mudanças aos municípios, seja pela criação de setores, pela mobilização de pessoas para lidar com a questão, ou mesmo pelo maior aporte de recursos que demandou aos

\section{Referências}

ARAÚJO, A. L. A. et al. Perfil da assistência farmacêutica na atenção primária do Sistema Único de Saúde. Ciênc. Saúde Colet., Rio de Janeiro, v. 13, supl., p. 611617, 2008.

CASTRO, S. H. R. Impacto desalocativo no orçamento público estadual em face de decisões judiciais. 2011. $50 f$. cofres municipais. Mas observou-se que, na maioria dos casos, o manejo das demandas por medicamentos se dava de forma totalmente destacada da AF. Não havia análise técnica dos pedidos, e muitas compras ocorriam de forma irregular. Não eram adotadas boas práticas para as atividades logísticas e a entrega dos medicamentos era feita sem orientação. Ficou clara a falta de estrutura dos municípios para a gestão das demandas.

No entanto, o que se pôde perceber é que os problemas ligados à $\mathrm{AF}$ existiam a priori nesses municípios e foram, possivelmente, determinantes da ocorrência de demandas judiciais. Falhas comuns da gestão da AF, de base, mostraram que as dificuldades advindas das demandas incidiram sobre municípios já fragilizados. A existência de listas de medicamentos não pactuados, muitas vezes maiores do que a pactuada, provocou aumento direto nas despesas. Em todos os municípios analisados foram verificadas enormes deficiências nas atividades da $\mathrm{AF}$ regular, falta de recursos humanos e falta de estrutura física para uma adequada disponibilização de medicamentos à população. Observou-se, ainda, que não houve subfinanciamento da AF nos municípios, mas indícios de gestão financeira por vezes pouco eficiente.

Neste sentido, a judicialização se coloca como um complicador da situação da gestão da AF em municípios brasileiros. Não se pode concluir, entretanto, que este fenômeno seja a causa de problemas, mas sim, em alguns casos, uma consequência das deficiências da própria gestão.

\section{Monografia (IV Prêmio Sof de Monografias). Belo Horizonte, 2011.}

CHIEFFI, A. L.; BARATA, R. C. B. Ações judiciais: estratégia da indústria farmacêutica para introdução de novos medicamentos Rev. Saúde Públ. São Paulo, v. 44, n. 3, p. 421-429, 2010. 
DE BERNARDI, C. L. B.; BIEBERBACH, E. W.; THOMÉ, H. I. Avaliação da assistência farmacêutica básica nos municípios de abrangência da $17^{\mathrm{a}}$ Coordenadoria Regional de Saúde do Rio Grande do Sul. Saúde Soc., São Paulo, v. 15, p. 73-83, 2006.

DELVECHIO, P. O Orçamento Público e o Processo de judicialização da saúde. 2010. 32f. Especialização (Orçamento Público) - Tribunal de Contas da União, Brasília, DF, 2010.

FIGUEIREDO, T. A.; PEPE, V. L. E.; OSORIO-DE-

CASTRO, C. G. S. Um enfoque sanitário sobre a demanda judicial de medicamentos. Physis, Rio de Janeiro, v. 20, n. 1, p. 101-118, 2010.

\section{INSTITUTO BRASILEIRO DE GEOGRAFIA E} ESTATÍSTICA (IBGE). Pesquisa Nacional por Amostra de Domicílios - 2009. 2009. Disponível em: < http://www.ibge. gov.br/home/estatistica/populacao/trabalhoerendimento/pnad2009/default.shtm>. Acesso em: 10 maio de 2011.

MACHADO, M. A. A. et al. Judicialização do acesso a medicamentos no Estado de Minas Gerais, Brasil. Rev. Saúde Públ., São Paulo, v. 45, n. 3, p. 590-598. 2011.

MARQUES, S. B.; DALLARI, S. G. Garantia do direito social à assistência farmacêutica no estado de São Paulo. Rev. Saúde Públ., São Paulo, v. 41, n. 1, p. 101-7, 2007.

\section{ORGANIZAÇÃO PAN-AMERICANA DA SAÚDE}

(OPAS). Avaliação da Assistência Farmacêutica no Brasil: estrutura, processo e resultados. Brasília, DF: Organização Mundial da Saúde; Brasília, DF: Ministério da Saúde, 2005.

PEPE, V. L. E. et al. A judicialização da saúde e os novos desafios da gestão da assistência farmacêutica. Ciênc. Saúde Colet., Rio de Janeiro, v. 15, n. 5, p. 2405-2414, 2010a.

Caracterização de demandas judiciais de fornecimento de medicamentos "essenciais" no Estado do Rio de Janeiro, Brasil. Cad. Saúde Pública, Rio de Janeiro, v. 26, n. 3, p. 461-471, 2010 b.
PEPE, V. L. E.; VENTURA, M.; OSORIO-DE-CASTRO, C. G. S. (Org.). Manual de indicadores de avaliação e monitoramento das demandas judiciais de medicamentos. Rio de Janeiro: Fundação Oswaldo Cruz, Escola Nacional de Saúde Pública Sergio Arouca, 2011, 56 p.

PEREIRA, J. R. et al. Análise das demandas judiciais para o fornecimento de medicamentos pela Secretaria de Estado da Saúde de Santa Catarina nos anos de 2003 e 2004. Ciênc. Saúde Colet. Rio de Janeiro, v. 15, supl. 3, p. 3551-60, 2010.

ROMERO, L. C. Judicialização das políticas de assistência farmacêutica: o caso do Distrito Federal. Brasília, DF: Senado Federal, Consultoria Legislativa, 2008, 48 p.

VIEIRA, F. S. Qualificação dos serviços farmacêuticos no Brasil: aspectos inconclusos da agenda do Sistema Único de Saúde. Rev. Panam. Salud Publica. Washington, DC, v. 24, n. 2, p. 91-100, 2008.

VIEIRA, F. S.; ZUCCHI, P. Distorções causadas pelas ações judiciais à política de medicamentos no Brasil. Rev. Saúde Pública, São Paulo, v. 41, n. 1, p. 214-22, 2007. Aplicações diretas para aquisição de medicamentos no Sistema Único de Saúde. Rev. Saúde Públ., São Paulo, v. 45, n. 5, p. 906-913, 2011.

\section{WORLD HEALTH ORGANIZATION (WHO).}

Drug and therapeutics committees: a practical guide Department of Essential Drugs and Medicines Policy. Geneva: WHO, 2003.

WRIGHT, J. T. C.; GIOVINAZZO, R. A. Delphi: uma ferramenta de apoio ao planejamento prospectivo. Caderno de Pesquisas em Administração, São Paulo, v. 1, n. 12 , p. $54-65,2000$.

Recebido para publicação em março de 2015

Versão final em outubro de 2015

Conflito de interesses: inexistente

Suporte financeiro: não houve 